\title{
Modernizing the Global Approximation Method of Posterior Probability Density for Oscillator Synchronization Purposes
}

\author{
Alexander V. Ryabov*, \\ Pavel A. Fedyunin and Maxim Y. Presnyakov \\ Military Training and Research Center of the Air Force \\ «Air Force Academy ft. Professor N.E. Zhukovsky and Y.A. Gagarin» \\ 54a Starykh Bol'shevikov Str., Voronezh, 394064, Russia
}

Received 16.02.2016, received in revised form 09.03.2016, accepted 06.03.2016

The article introduces modernizing the global approximation method of the posterior probability density function in the implementation of phase-locked loop algorithms which improves the accuracy of clock synchronization in telecommunication networks, and reduces the time of entering into synchronism.

Keywords: clock synchronization; phase-locked loop; the global approximation; filtering; synchronism.

Citation: Ryabov A.V., Fedyunin P.A., Presnyakov M.Y. Modernizing the global approximation method of posterior probability density for oscillator synchronization purposes, J. Sib. Fed. Univ. Eng. technol., 2016, 9(4), 462-469. DOI: 10.17516/1999-494X-2016-9-4-462-469.

(C) Siberian Federal University. All rights reserved

* Corresponding author E-mail address: ryalvy@mail.ru 


\title{
Модернизация метода интегральной аппроксимации апостериорной плотности вероятности \\ в задачах тактовой синхронизации генераторов
}

\author{
А.В. Рябов, П.А. Федюнин, М.Ю. Пресняков \\ ВУНЦ ВВС «ВВА им. проф. Н.Е. Жуковского и Ю.А. Гагарина» \\ Россия, 371600, Воронеж, ул. Старых Большевиков, $54 a$
}

\begin{abstract}
Предложена модернизация метода интегральной аппроксимации апостериорной плотности распределения вероятности при реализации алгоритмов фазовой автоподстройки частоты, позволяющая повысить точность тактовой синхронизации в телекоммуникационных сетях и уменьшить время вхождения в синхронизм.
\end{abstract}

Ключевые слова: тактовая синхронизация, фазовая автоподстройка частоты, интегральная аппроксимация, фильтращия, синхронизм.

\section{Introduction}

In modern telecommunication networks the quality of data transmission largely depends upon the accuracy of clock synchronization. Furthermore, in high-speed networks in order to provide the minimum synchronization time at the start and the minimum time of a sync recovery process in case of a breakdown, the synchronization requirements are becoming more severe. In addition, if the sync mode is continuous and automated, the high stability of the network element synchronism is required.

Solving oscillator clock synchronization problems in telecommunication networks is normally based upon designing a phase-locked loop system of oscillator frequency at the receiver site. Moreover, for a phase-locked loop implementation the phase of the received signal should be estimated [1].

Let the signal produced by a reference oscillator at the transmitter site of a telecommunication channel, be a harmonic motion

$$
S(t, \varphi)=A \sin \left(\omega_{0} t+\varphi(t)\right) .
$$

where $-\pi \leq \varphi(t) \leq \pi$ denotes a random Wiener phase, defined by the equation:

$$
d \varphi(t) / d t=n_{\varphi}(t)
$$

where $n_{\varphi}(t)$ is the additive white Gaussian noise (AWGN) determined by the internal reference oscillator noise, with zero mean and the correlation function $\mathrm{M}\left[n_{\varphi}\left(t_{1}\right) \cdot n_{\varphi}\left(t_{2}\right)\right]=\left(N_{\varphi} / 2\right) \cdot \delta\left(t_{2}-t_{1}\right)$.

At the receiver site of the communication channel there is a mixture of desired signal and noise:

$$
\xi(t)=S(t, \varphi)+n_{0}(t)
$$

where $n_{0}(t)$ is the AWGN determined by external noise, with the expected value with zero mean and the correlation $\mathrm{M}\left[n_{0}\left(t_{1}\right) \cdot n_{0}\left(t_{2}\right)\right]=\left(N_{0} / 2\right) \cdot \delta\left(t_{2}-t_{1}\right)[1]$.

In order to create a self-oscillator phase-locked loop system at the receiver site it is necessary to obtain an optimal filtering algorithm for a received signal phase $\varphi(t)$. Let the highest posterior probability

$$
-463-
$$


density of a random signal phase and the minimum mean square filtering error be optimality criteria. Then it is necessary to solve the problem of a received signal phase filtering for a small signal-to-noise ratio.

Since the Equation in (3) is nonlinear in relation to phase $\varphi(t)$, it is essential to solve the task of nonlinear Wiener phase filtering. In theory finding the solution is based upon solving Stratonovich SDEs.

$$
\begin{gathered}
\partial p(t, \varphi) / \partial t=0.25 N_{\varphi} \partial^{2} p(t, \varphi) / \partial \varphi^{2}+\left[F(t, \varphi)-\int_{-\pi}^{\pi} F(t, \varphi) p(t, \varphi) d \varphi\right] p(t, \varphi) ; \\
F(t, \varphi)=2 N_{0}^{-1}(\xi(t)-S(t, \varphi))^{2} \approx 2 A \xi(t) N_{0}^{-1} \sin \left(\omega_{0} t+\varphi(t)\right) .
\end{gathered}
$$

Nevertheless, an accurate solution to the nonlinear filtering equations can be obtained in few cases $[1,2]$. Therefore, it is essential to focus upon developing approximate nonlinear filtering equation solutions.

The aim of this paper is to compare and analyze the existing solution methods of nonlinear Stratonovich SDEs and to suggest modernizing the general approximation method of the posterior probability density function, which increases the accuracy of signal phase filtering and reduces the time of entering into synchronism.

\section{Analysis of the nonlinear filtering equation solution techniques for a random received signal phase}

The most common technique is synthesizing algorithms of nonlinear filtering using the ways of approximation to the posterior probability density $p(t, \varphi)$, some function $p(t, \varphi, \boldsymbol{\alpha})$ belonging to the parameterized class $\boldsymbol{\alpha} \in \boldsymbol{\Psi}[1]$.

The techniques of approximating to the posterior probability density function can be subdivided into two categories: the local density approximation and the global approximation [1].

In local approximation approximate filtering algorithms are obtained due to an accurate solution approximation for a small set of assessment values $\hat{\varphi}(t)$ of the filtering parametric variable. This approach allows to obtain functional algorithms in special cases.

Normally the local density approximation is based upon replacing a probability density function $p(t, \varphi)$ by a probability density function of a normal distribution $p(\hat{\varphi}(t) R(t))$, where $-\pi<\hat{\varphi}(t)<\pi$, $0<R(t)<\infty$.

Let us look at some quasi optimal algorithms based upon this approach.

1. The extended Kalman filter (EKF)

This quasi optimal algorithm is based on reducing the initial nonlinear filtering task to a linear due to expanding nonlinear functions which are part of observation equations and signal message equations in a Taylor series a close vicinity of the estimation value $\hat{\varphi}[1]$.

Consequently, the algorithm of phase filtering is as follows:

$$
\begin{aligned}
& d \widehat{\varphi} / d t=2 A \xi(t) R(t) N_{0}^{-1} \cos \left(\omega_{0} t+\widehat{\varphi}(t)\right) \\
& d R(t) / d t=N_{\varphi} / 2-A^{2} R^{2}(t) N_{0}^{-1} .
\end{aligned}
$$

2. Local approximation techniques based on finding approximate solutions to Stratonovich SDEs.

$$
-464-
$$


To obtain a solution the posterior probability density logarithm $p(t, \varphi)$ is to be expanded in a Taylor series near the tentative estimate $\hat{\varphi}$ corresponding to the highest posterior probability density [1]. The equations of phase filtering estimation and mean square filtering error can be represented in the following way:

$$
\begin{aligned}
& d \hat{\varphi} / d t=2 A \xi(t) R(t) N_{0}^{-1} \cos \left(\omega_{0} t+\hat{\varphi}(t)\right) \\
& d R(t) / d t=N_{\varphi} / 2-A^{2} R^{2}(t) \xi(t) N_{0}^{-1} \sin \left(\omega_{0} t+\hat{\varphi}(t)\right) .
\end{aligned}
$$

The main drawback of local approximation techniques for the posterior probability density is the fact that they can only be applied when filtering errors are minimal, i.e. the SNR is high, and a good approximation of the main filtering equation is required only in a close vicinity of a filtering parametric variable $\hat{\varphi}(t)$. This drawback makes it impossible to apply this method of approximation to solving many important practical problems [2].

In global approximation approximate filtering algorithms are obtained due to approximating an accurate solution $p(t, \varphi)$ to some probability density function $p(t, \varphi, \boldsymbol{\alpha})$ chosen in accordance with physical representations. The solution is sought within the whole range of possible values of the filtering parametric variable $\varphi$. A certain integral criterion is required, which is especially important for a small signal-to-noise ratio [2]. The minimum Kullback-Leibler divergence can be regarded as such a criterion, as it ensures minimal information loss due to the posterior probability density approximation [3]. The advantage of this criterion consists in emphasizing the tails of the distribution and in increasing their significance. According to this criterion for a fitting probability density function a parametric variable $\hat{\boldsymbol{\alpha}}$ is chosen in such a way that it minimizes the integral [3]:

$$
\widehat{\boldsymbol{\alpha}}(t)=\min _{\alpha}^{-1}\left\{-\int_{-\infty}^{\infty} p(t, \varphi) \ln [p(t, \varphi, \boldsymbol{\alpha}) / p(t, \varphi)] d \varphi\right\} .
$$

Here is the necessary condition for the minimum Kullback-Leibler divergence [3]:

$$
\int_{-\infty}^{\infty} \partial[p(t, \varphi, \boldsymbol{\alpha})] / \partial \boldsymbol{\alpha} p(t, \varphi) \ln d \varphi=0
$$

Then the general equation for parametric variables of the fitting probability density function can be represented as follows:

$$
\frac{\partial \boldsymbol{\alpha}}{\partial t}=\frac{\mathrm{M}\left[\mathrm{L}^{+}\left[\frac{\partial \ln \{p(t, \varphi, \boldsymbol{\alpha})\}}{\partial \boldsymbol{\alpha}}\right]\right]+\mathrm{M}\left[\frac{\partial \ln \{p(t, \varphi, \boldsymbol{\alpha})\}}{\partial \boldsymbol{\alpha}}\right] \cdot F(t, \varphi)}{\mathrm{M}\left[-\frac{\partial^{2} \ln \{p(t, \varphi, \boldsymbol{\alpha})\}}{\partial \boldsymbol{\alpha} \partial \boldsymbol{\alpha}^{\mathrm{T}}}\right]},
$$

where $\mathrm{M}[\cdot]$ is a mathematical expectation; $\mathrm{L}^{+}[\cdot]$ is an operator that is the backward dual Fokker-PlanckKolmogorov Equation [3]

Let us look at the two known types of general approximation $[2,4]$.

1. The approximating probability density is found in the normal probability density class $p(\hat{\varphi}(t) R(t))[2,4]$ : 


$$
p(\widehat{\varphi}(t) R(t))=1 / \sqrt{2 \pi R(t)} \exp \left\{(\varphi(t)-\hat{\varphi}(t))^{2} / 2 R(t)\right\} .
$$

In this case the random Wiener phase filtering algorithm will be the following [2]:

$$
\begin{aligned}
& d \hat{\varphi} / d t=2 A \xi(t) R(t) N_{0}^{-1} \exp \{-R(t) / 2\} \cos \left(\omega_{0} t+\widehat{\varphi}(t)\right) ; \\
& d R(t) / d t=N_{\varphi} / 2-2 A R^{2}(t) \xi(t) N_{0}^{-1} \exp \{-R(t) / 2\} \sin \left(\omega_{0} t+\hat{\varphi}(t)\right) .
\end{aligned}
$$

2. The T-distribution approximation is [2]:

$$
p(t, \varphi, \boldsymbol{\alpha})=1 / \sqrt{2 \pi I_{0}(\Lambda)} \exp \left\{\Lambda \cos \left(\varphi-m_{\varphi}(t)\right)\right\}
$$

where $I_{0}(\Lambda)$ is a modified zero order Bessel function of imaginary argument; $\boldsymbol{\alpha}=\left\{m_{\varphi}(\mathrm{t}), \Lambda(\mathrm{t})\right\}$ are distribution parameters, $\Lambda \geq 0 ; \varphi \in\left[m_{\varphi}-\pi, m_{\varphi}+\pi\right]$.

This probability density function approaches the normal law of distribution with period $2 \pi$ recurrence if the $\Lambda$ value is high; if $\Lambda \rightarrow 0$, it converges into uniform probability density.

Then the random Wiener phase filtering algorithm may be written in the following way [2]:

$$
\begin{aligned}
& d m_{\varphi}(t) / d t=2 A \xi(t) N_{0}^{-1} / \Lambda(t) \cos \left(\omega_{0} t+m_{\varphi}(t)\right) \\
& d \Lambda(t) / d t=-N_{\varphi} / 4 f_{1}(\Lambda)+2 A \xi(t) N_{0}^{-1} \sin \left(\omega_{0} t+m_{\varphi}(t)\right),
\end{aligned}
$$

where

$$
\begin{aligned}
& 1 / \Lambda(t)=\int_{-\infty}^{\infty}\left(\varphi-m_{\varphi}(t)\right)^{2} p(t, \varphi, \boldsymbol{\alpha}) d \varphi \\
& f_{1}(\Lambda)=I_{1}(\Lambda) / I_{0}(\Lambda)\left(1-I_{1}(\Lambda) /\left(\Lambda(t) I_{0}(\Lambda)\right)-\left[I_{1}(\Lambda) / I_{0}(\Lambda)\right]^{2}\right)^{-1}
\end{aligned}
$$

$I_{1}(\Lambda)$ is a modified zero order Bessel function of imaginary argument.

If we look at the dependence between the filtering error variance and the signal-to-noise ratio for the algorithms mentioned above,

$$
q=4 A^{2} /\left(N_{\varphi} \cdot N_{0}\right)
$$

we will see that for a large signal-to-noise ratio the accuracy of all the algorithms will be close to the potential accuracy.

For a small signal-to-noise ratio the accuracy of filtering algorithms based upon the abovementioned approximation techniques will be considerably lower than the potential accuracy.

The most accurate filtering algorithm from the ones mentioned above can be implemented with the help of the general T-approximation (17), (18) [2]. Nevertheless, this algorithm is lengthy and hard to put into practice, whereas the quasi optimal algorithm of local approximation is the least accurate for the small value domain of the signal-to-noise ratio [2].

With the signal-to-noise ratio $q \rightarrow 0$, in accordance with the accepted criterion, the value of the variances under consideration approaches the limit

$$
\lim _{q \rightarrow 0} M\left\{[\varphi-\hat{\varphi}(t)]^{2}\right\}=\pi^{2} / 3
$$


When there is no desired signal, the filtering error of all the algorithms is uniformly distributed within the $(-\pi, \pi)$ interval.

Furthermore, the abovementioned algorithms do not meet the minimal time of acquiring synchronization requirement which is essential for developing phase-locked loop systems.

Consequently, the analysis of the algorithms mentioned above has shown that they are insufficient for solving the problem.

\section{Modernizing the global approximation method of posterior probability density}

In order to solve the problem of filtering a received oscillation phase with a small signal-to-noise ratio it is appropriate to use the modernized general approximation technique based upon using the AWGN.

Let us represent the filtering error variance $R(t)$ of the truth posterior probability $p(t, \varphi)$ density of a random Wiener filter signal phase as:

$$
1 / R(t)=1 / R 1(t)+3 / \pi^{2},
$$

where $R 1(t)$ is the filtering error variance component of approximating posterior probability density without considering the constant component.

Now we search for the approximating probability density in the class of normal probability density distribution of type (13) with regard to the Expression in (23).

$$
\begin{aligned}
& p(\varphi, m(t) G 1(t))=\sqrt{G 1(t) / 2 \pi} \exp \left\{-G 1(t)\left(\varphi-m_{\varphi}(t)\right)^{2} / 2\right\} \\
& G 1(t)=1 / R 1(t)=G(t)-3 / \pi^{2}
\end{aligned}
$$

where $0 \leq G 1(t) \leq \infty$.

Then using Functions (24) and (25) we seek the approximate received signal phase $\varphi(t)$ filtering algorithm:

$$
\begin{aligned}
& d m_{\varphi}(t) / d t=2 A \xi(t) N_{0}^{-1} /\left(G(t)-3 / \pi^{2}\right) \cos \left(\omega_{0} t+\hat{\varphi}(t)\right) \\
& d G(t) / d t=-N_{\varphi} / 2\left(G(t)-3 / \pi^{2}\right)^{2}+2 A \xi(t) N_{0}^{-1} \sin \left(\omega_{0} t+\hat{\varphi}(t)\right)
\end{aligned}
$$

Figures 1 and 2 show the dependency of the $R(t)=d^{2}$ variance and the inverse function of the $1 / d^{2}$ variance of a stationary filtering error on the signal-to-noise ratio $q$ obtained due to simulation modeling of algorithms (4), (7), (18) and (27).

In addition, the $d^{2}$ function corresponds to an exact solution (4), the $d_{1}^{2}$ function corresponds to the extended Kalman filter (7); the $d_{2}{ }^{2}$ function is a T-approximation (18); the $d_{3}^{2}$ function is the modernized general approximation (28).

In Fig. (2) and (3) the $d^{2}$ function and the $d_{3}^{2}$ functions coincide.

As we can see from the simulation results analysis, the modernized technique of approximating to the general posterior probability density suggested by the authors helps to obtain the random phase $\varphi(t)$ filtering algorithm of the received signal that coincides with an accurate solution. Therefore, in order to solve the problem under consideration, this algorithm (26), (27) can be regarded as an optimal one. 


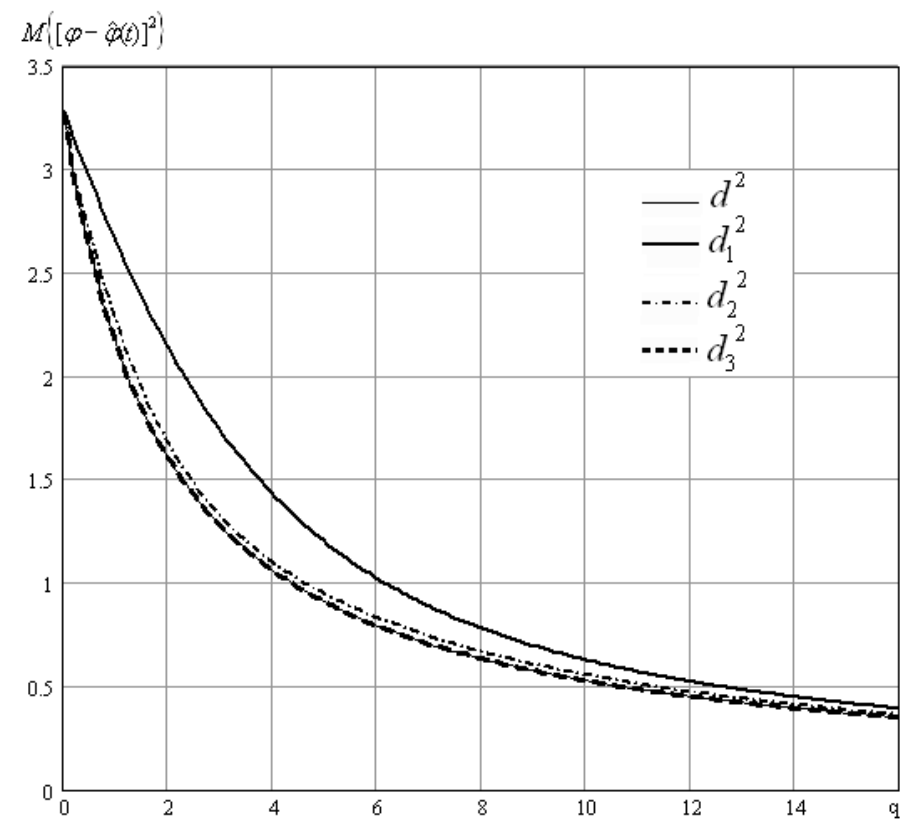

Fig. 1. Dependency of the $R(t)=d^{2}$ variance of a stationary filtering error on the signal-to-noise ratio $q$

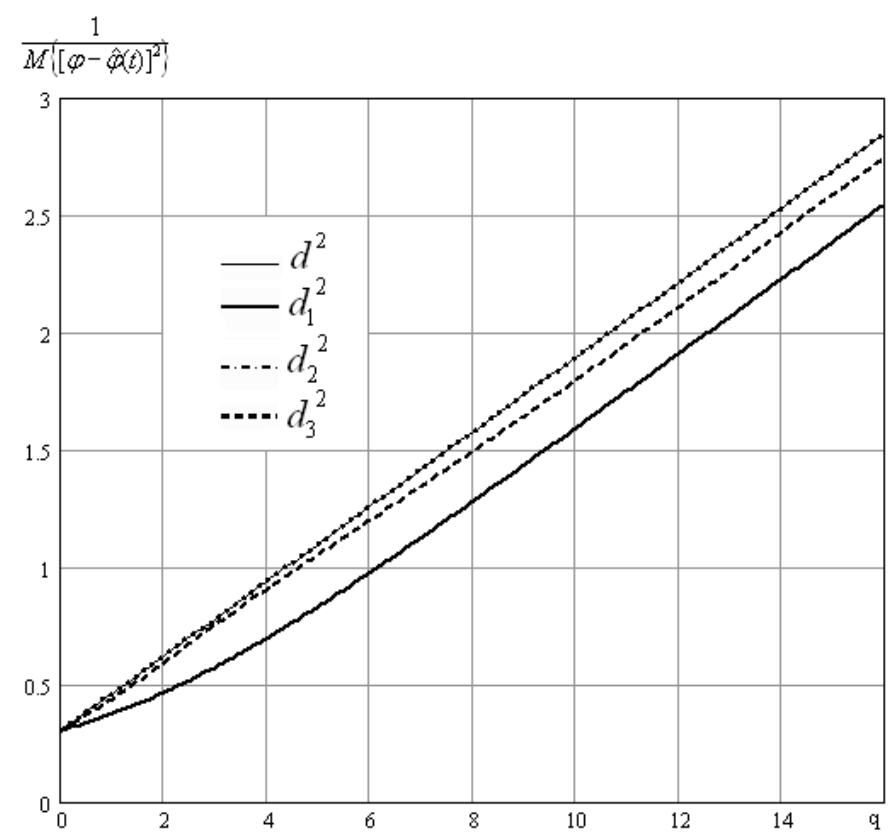

Fig. 2. Dependency of the inverse function of the $1 / d^{2}$ variance of a stationary filtering error on the signal-to-noise ratio $q$ 
Fig. 2 shows that there is a line coming out from the point $3 / \pi^{2}$ corresponding to an accurate solution. For the small value domain of the signal-to-noise ratio the dependency graphs of the inverse function of the $1 / d^{2}$ variance of a stationary filtering error on the signal-to-noise ratio $q$ obtained due to algorithms (7) and (18) have nonlinear parts.

Consequently, in order to estimate the filtering algorithm optimality of a random signal phase it is worthwhile using the inverse function, but not the filtering error variance itself. Then a linear function should be the optimality criterion.

Algorithms (18) and (27) differ due to their multipliers with $N_{\varphi} / 2$ as part of the first summand.

We can modernize the extended Kalman filter algorithm represented by Expressions (6) and (7) in the same way. After being modernized it will look as follows:

$$
\begin{aligned}
& d \hat{\varphi} / d t=2 A \xi(t) N_{0}^{-1} /\left(G(t)-3 / \pi^{2}\right) \cos \left(\omega_{0} t+\hat{\varphi}(t)\right) ; \\
& d G(t) / d t=-N_{\varphi} / 2\left(G(t)-3 / \pi^{2}\right)^{2}+A^{2} / N_{0},
\end{aligned}
$$

where $G(t)=1 / R(t)$.

\section{Conclusions}

We have suggested the way of modernizing the general approximation method of posterior probability density for a random received signal phase, due to this modernization distribution limits and the limit of the function are taken into consideration.

The filtering algorithms for a random received signal phase that we have developed due to the obtained approximation meet the optimality criterion for both high and small signal-to-noise ratio. These algorithms make it possible to implement the phase-locked loop system in High Precision Oscillators as well as to gain the time of entering into synchronism, which is vitally important for developing high-speed telecommunication networks.

\section{References}

[1] Тихонов В.И., Харисов В.Н. Статистический анализ и синтез радиотехнических устройств и систем. М.: Радио и связь, 1991, 608c. [Tikhonov V.I., Harisov V.N. Statistic Analysis and Synthesis of Radio Equipment and Systems. Moscow, Radio and Svyaz, 1991, 608 p. (in Russian)]

[2] Харисов В.Н., Федоров А.И. Синтез алгоритмов нелинейной фильтрации на основе интегральной аппроксимации апостериорной плотности вероятности. Научно-методические материалы по статистической радиотехнике. М.: ВВИА им. Жуковского, 1985, 161-166 [Harisov V.N., Fedorov A.I. Synthesizing Nonlinear Filtering Algorithms Based on General Approximation to Posterior Probability Densityю. Research and Methodology Study Materials in Statistical Radio Technology. Moscow: Zhukovsky Air Force Engineering Academy, 1985, 161-166. (in Russian)]

[3] Abramowitz M, Stegun I.A. Handbook of mathematical functions with formulas, graphs, and mathematical tables. Washington, D.C., 1970. 1048 p.

[4] Рубан А.И. Дисперсионные характеристики статистических моделей стохастических объектов. Журнал СФУ. Техника и технологии, 2009, 2(1), 88-99. [Ruban A.I. Dispersion characteristics of the statistical models of stochastic objects, J. Sib. Fed. Univ. Eng. technol., 2009, 2(1), 88-99 (in Russian)] 